\title{
Muscle spindle activity in man during voluntary fast alternating movements ${ }^{1}$
}

\author{
K.-E. HAGBARTH ${ }^{2}$, G. WALLIN, AND L. LÖFSTEDT \\ From the Department of Clinical Neurophysiology, University Hospital, Uppsala, Sweden
}

\begin{abstract}
SYNOPSIS Single unit activity in primary spindle afferent nerve fibres from finger and foot flexors was recorded with tungsten microelectrodes inserted into the median and peroneal nerves of healthy subjects. During voluntary fast alternating finger and foot movements, simulating the tremor of Parkinsonism, two types of discharges were seen in the Ia afferent fibres: (1) stretch responses occurring during the flexor relaxation phases, and (2) discharges occurring during the flexor contraction phases. Contrary to the stretch responses the spindle contraction discharges could be eliminated by a partial lidocaine block of the muscle nerve proximal to the recording site, indicating that they resulted from fusimotor activation of intrafusal fibres. On the basis of the temporal relations between the beginning and end of individual EMG-bursts, the start of the spindle contraction discharges and the latency of the stretch reflex in the muscles concerned, the following conclusions were drawn: the recurrent extrafusal contractions in movements of this type are initiated by the fast direct alpha route, but individual contraction phases generally last long enough to be influenced subsequently by the coactivated fusimotor loop through the spindles. It is postulated that this gamma loop influence during alternating movements helps to keep flexor and extensor muscles working in a regular reciprocal fashion with contractions adjusted in strength to the external loads.
\end{abstract}

As shown in previous recordings from single muscle spindle afferent nerve fibres in man, the impulse frequency in these fibres increases during voluntary isometric contractions of the muscle in which the spindles are located (Hagbarth and Vallbo, 1969; Vallbo, 1970, 1971). According to Wallin et al. (1973) this contraction discharge from the spindles, as seen in multi-unit recordings from the muscle nerve, is reduced after a partial lidocaine nerve block affecting primarily the fusimotor fibres. These findings agree with the notion that voluntary contractions are organized according to the principle of alphagamma coactivation, the extrafusal muscle contractions being accompanied by a fusimotor activation of the spindles counteracting the mechanical unloading effect on these organs caused by the shortening of neighbouring extrafusal muscle fibres.

However, in various types of coordinated

1 The investigation was supported by the Swedish Medical Research Council (Project no. B75-04X-2881-06A).

2 Reprint requests: Dr K.-E. Hagbarth, at above address. (Accepted 18 February 1975.) skilled motor acts individual muscles can work in many different ways - for example, as stabilizers, load-carriers, steering-organs for slow movements, accelerators and decelerators in fast movements of the ballistic type, etc., and it is still unknown whether the principle of alphagamma coactivation applies to all these various types of motor performance. The present study deals with the problem of how the fusimotor system and the stretch reflex operates in voluntary fast alternating finger- and foot movements, in which the prime movers work predominantly as decelerators and accelerators. In a succeeding report, abnormal involuntary movements of the alternating type-clonus and tremor of Parkinsonism-are subjected to a similar analysis.

\section{METHODS}

MATERIAL Nineteen experiments were made on 11 healthy adults varying in age between 27 to 50 years. The nerve recordings were made either from median muscle nerve fascicles at the elbow level supplying the long finger flexor muscles (nine experiments), 
from peroneal muscle nerve fascicles at the level of the fibular head supplying the anterior tibial or long peroneal muscles (seven experiments), or from tibial muscle nerve fascicles in the popliteal fossa supplying the triceps surae muscles (three experiments). The criteria that the electrode tip was located in a muscle nerve bundle were as follows: (1) no signs of neural activity in response to skin strokings or light tactile skin stimuli, but distinct multi-unit discharges in response to local muscle belly taps, passive muscle stretch, or isometric voluntary contractions; (2) localized muscle twitches and absence of radiating skin paraesthesiae in response to electric test shocks delivered through the electrode tip. In the Results section an account is given of the number of mechanoreceptive units collected and the methods of identifying muscle spindle receptors.

MEASUREMENTS OF JOINT ANGLE AND TORQUE IN ACTIVE AND PASSIVE MOVEMENTS When recording from nerves to finger flexor muscles the subject was sitting with his outstretched arm resting on a support that held the elbow and wrist joint in an extended position with the metacarpophalangeal joints of fingers 2-5 placed over the pivot of a rotating plate to which the fingers were fixed with extended interphalangeal joints. Thus, the movements were restricted as far as possible to the metacarpophalangeal joints and the subject could by finger flexions and extensions turn the plate back and forth in a horizontal plane at desired speed. Sometimes a rubber band was attached to the plate to add extra resistance to the finger flexion movements. The torque, given in metre-newtons $(\mathrm{Nm})$, was measured with a strain gauge mounted on the rotating plate and angular position was measured by a potentiometer mounted on the pivot. The potentiometer was calibrated to indicate zero degrees when the metacarpophalangeal joints were fully extended. The plate could be fixed in any desired position to make observations under isometric conditions and it could also be driven either manually or by an electrically controlled hydraulic device in passive steps of variable speed and amplitude.

When recording from nerves to ankle flexor or extensor muscles the subject was lying on his side with the knee extended and the foot attached to the rotating plate, thus restricting movement to plantarand dorsiflexion of the ankle joint. In these tests the potentiometer was calibrated to indicate zero degrees when the sole was at right angles to the anterior edge of the tibia. The potentiometer signals were displayed in such a way that downward deflections always indicated stretching of the muscle from which the receptor discharges originated, whereas increased tension of the receptor bearing muscle was always displayed as an upward deflection in the torque record.

NERVE RECORDING TECHNIQUE The recording technique and equipment were essentially the same as described previously (Hagbarth et al., 1970). Thus, the recording electrodes were coated tungsten needles with shaft diameter of $0.2 \mathrm{~mm}$, tip diameter of 1-5 $\mu \mathrm{m}$, and impedance of $50-100 \mathrm{k} \Omega$ measured at $1 \mathrm{kHz}$. A tungsten needle with a $5 \mathrm{~mm}$ long bare tip inserted in the vicinity of the intraneural electrode served as reference. The recording electrode was manually directed toward the nerve while single electric pulses were applied through the electrode tip. When minimal shocks gave contractions in an appropriate muscle, stimulation was discontinued and very small movements of the electrode position were made in the search for single unit potentials from intramuscular mechanoreceptors (see Results section).

The amplifiers, filters, and noise reducing circuits have been described previously (Hagbarth et al., 1970). An instantaneous frequency meter of the N type described by Green (1967) was often used for analysis of single unit activity.

ELECTROMYOGRAMS AND ELECTRICALLY INDUCE尺̊̊ MUSCLE TWITCHES The electromyograms (EMG $\overrightarrow{0}$ were recorded bipolarly either with surface electrodes or with needles similar to the nerve reference electrodes placed 2-3 cm apart. Coaxial needle electrodes were also used occasionally. When recording neural responses to twitch contractions, the receptor bearing muscle was electrically stimulated either through the bipolar needle EMG electrodes or through a similar pair of needles inserted nearby in the muscle belly. $\overline{0}$ Care was taken to place the intramuscular electrodes in that particular muscle or muscle portion from which neural afferent discharges could be elicited by local taps and where twitches occurred in response to intraneural electric stimuli.

STORING AND DISPLAY SYSTEMS Nerve action poten- $-\frac{3}{3}$ tials, electromyograms, torque- and goniometer sig- 응 nals were displayed on a 549 Tektronix oscilloscope $₹$ and recorded by a multichannel tape recorder 0 (Precision Instrument type 6208). An audio amplifier and a loudspeaker were used to monitor nerve action potentials. When the tapes were replayed the signals were photographed with a Polaroid camera $\sigma$ from the oscilloscope.

\section{RESULTS}

IDENTIFICATION AND GENERAL CHARACTERISTICS OF PRIMARY MUSCLE SPINDLE AFFERENT FIBRES 
Altogether 73 nerve units were encountered from low threshold muscle mechano-receptors, exhibiting short latency responses to weak tendon or muscle belly taps. Twenty of these units were insufficiently tested to permit any further identification. Five units with extreme dynamic sensitivity to taps but unresponsive to maintained pressure or stretch were classified as Pacinian corpuscles, whereas six of the stretch receptor units, which fired during the rising phase of electrically induced muscle twitches, were denoted as Ib afferents. Five units considered to be group II spindle afferent fibres, showed unloading responses to electrically-induced twitches and a static discharge which increased with passive elongation of the muscle relatively independent of the speed of movement. Thirty-seven units were classified as primary muscle spindle afferent fibres (Ia) according to the following criteria: (1) dynamic responses to passive muscle stretch increasing with the speed of the

TABLE

PRIMARY MUSCLE SPINDLE AFFERENTS AND LOCALIZATION OF RECEPTORS

\begin{tabular}{lrr}
\hline & $\begin{array}{c}\text { Ja fibres } \\
\text { sampled }\end{array}$ \\
\hline Median nerve & & \\
$\quad$ Superficial and deep finger flexors & 13 & 8 \\
$\quad$ Flexor carpi and palmaris longus & 4 & 0 \\
Peroneal nerve & & \\
$\quad$ Anterior tibial & 11 & 7 \\
Long peroneal & 3 & 0 \\
Tibial nerve & & \\
Medial and lateral gastrocnemius & 4 & 1 \\
Soleus & 2 & 0 \\
\hline & 37 & 16 \\
\hline
\end{tabular}

movement, (2) silence during passive muscle shortening, (3) a pause during the contractionand a discharge during the relaxation phase of electrically-induced muscle twitches (el-twitch tests).

The Table shows the number of primary muscle spindle afferent fibres sampled in different nerves, and the localization of the receptors as judged primarily by tapping- and el-twitch tests.
The figures in the Table that are italic indicate the number of Ia afferent fibres studied during voluntary fast alternating movements or isometric alternating muscle contractions.

Figures 1-3 illustrate some general discharge characteristics of the Ia afferent fibres. During passive alternating joint movements their firing rate was always maximal in the early or middle part of the stretch phases, increasing with the speed of the movement. Some units, like the finger flexor unit illustrated in Fig. 1A required angular movements of comparatively high speed $\left(6^{\circ} / \mathrm{s}\right)$ and amplitude $\left(10^{\circ}\right)$ to become

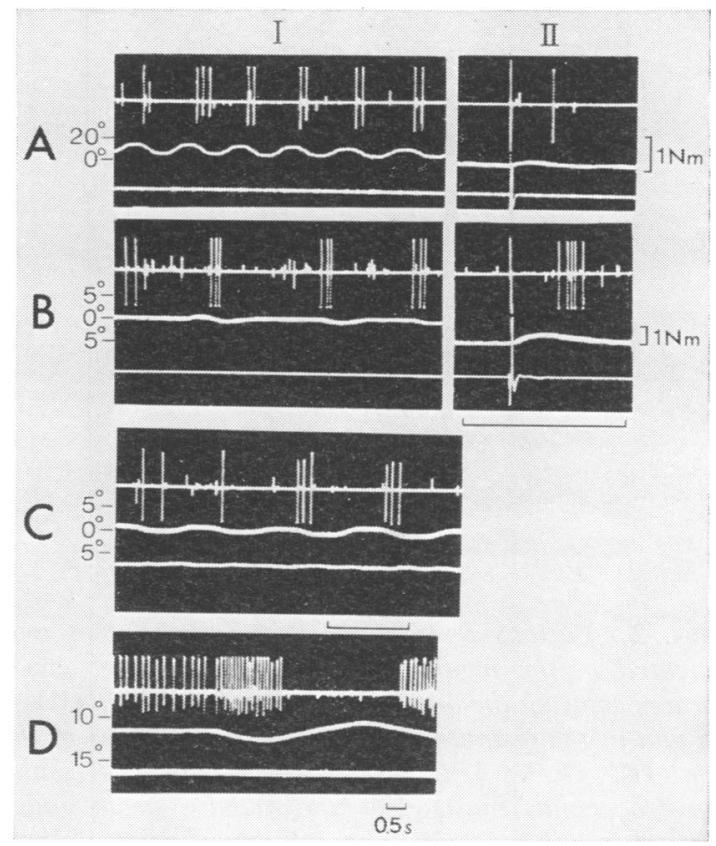

FIG. 1 Examples of stretch discharges in Ia afferent units during passive alternating finger and foot movements (I) and electrically elicited twitches (II) of the receptor bearing muscle. Units $\mathrm{B}$ and $\mathrm{C}$ located in the anterior tibial muscle, unit $\mathrm{A}$ in the superficial finger flexor and unit $\mathrm{D}$ in the gastrocnemius muscle. Upper trace: nerve record. Middle trace in I: angle in the metacarpophalangeal or ankle joints. $0^{\circ}$ denotes completely extended fingers and foot at right angle to the tibia respectively. In this and all other Figures muscle stretch is drawn downwards. Middle trace in II: torque. Bottom trace: EMG in the finger flexor (A), anterior tibial (B) and (C), and gastrocnemius muscle (D) respectively. 
activated during the stretch phases, whereas others, like the anterior tibial unit in Fig. 1B, responded to angular movements of less than $1^{\circ}$. The relative stretch sensitivity of individual spindle receptors as tested in this way remained largely uninfluenced by changes in the attentive state of the subject and by active movements in remote body segments. However, any manoeuvre that changed the mean length of the

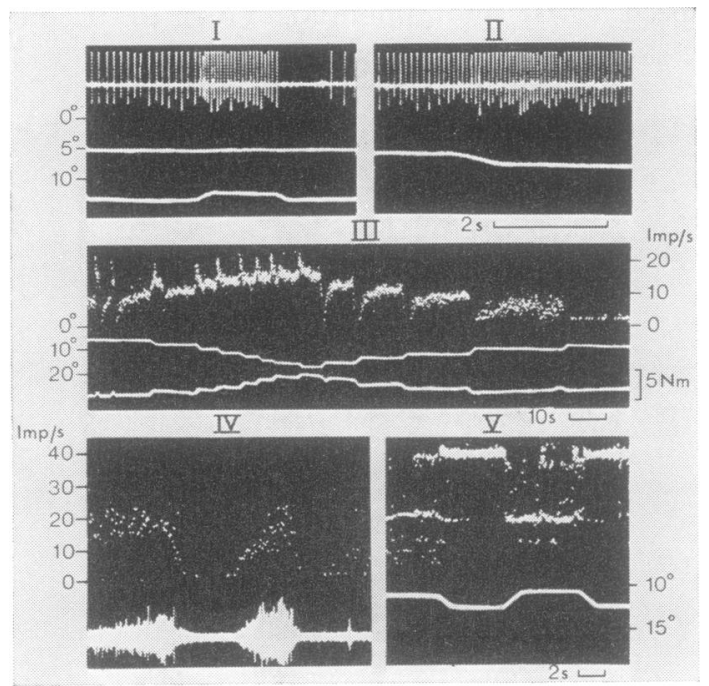

FIG. 2 Various tests applied to a Ia afferent unit located in the medial gastrocnemius muscle, upper traces showing the original nerve record (I) and (II) or a plot of the instantaneous discharge frequency of the unit (III, IV, V). I: Local pressure applied over medial gastrocnemius muscle as indicated by strain gauge signal in bottom trace. The ankle joint passively held in $5^{\circ}$ dorsiflexion (middle trace) with calf muscles remaining relaxed. II: calf muscle stretch by passive foot dorsiflexion. III: length changes of relaxed calf muscles induced by passively moving ankle joint in steps of about $3^{\circ}$ as indicated by middle trace. Torque changes shown in bottom trace (initial two deflections induced by temporarily applied local pressure on muscle belly as in (I). IV: Isometric voluntary calf muscle contractions of moderate strength as indicated by bottom EMG trace. Ankle joint in position $0^{\circ}$. V: $A$ mechanical vibrator beating at $40 \mathrm{~Hz}$ with an amplitude of $1.5 \mathrm{~mm}$ attached to the Achilles tendon while passively changing the length of the calf muscles. Note that unit is driven either at 40 or 20/s depending on ankle joint position. receptor bearing muscle or activated its motor units tended to influence the spindle responses. As the 'background' static spindle discharge rose with increasing mean muscle length so the absolute number of impulses per stretch phase was also enhanced. On the other hand, the shortening phases were usually accompanied by a total arrest of the spindle discharge, independent $\stackrel{0}{\stackrel{D}{a}}$ of the static firing level. Thus, for passive alternating movements with a given speed and amplitude the contrasts in impulse density during stretch and shortening phases increased with increasing mean muscle length. The gastrocnemius spindle shown in Fig. 1D was initially stretched $\vec{\circ}$ to a level where its static firing rate was about 5-6 imp/s. In response to alternating ankle ${ }_{\sigma}^{\omega}$ movements of a few degrees, so slow that they were not perceived by the subject, the steady state discharge was converted into high frequency $\underset{\infty}{\omega}$ stretch discharges, alternating with periods of $v$ complete silence during the shortening phases. N Another way of studying the dynamic and $\mathrm{O}$ static stretch sensitivity of individual spindle afferents is illustrated in Fig. 2, III. The instans taneous frequency plot shows how a spindle 兽 the relaxed gastrocnemius muscle responds when $\vec{\theta}$ the ankle is moved in steps from one level of ज dorsiflexion to another, each step movement of $3^{\circ}$ lasting about $1.0 \mathrm{~s}$. Units with dynamic stretch discharges and unloading pauses like those seen in the Figure were classified as Ia afferents, whereas units with no or only a mild $\stackrel{\mathbb{Q}}{\circ}$ tendency to such 'overshooting' were con- $\overrightarrow{\overrightarrow{0}}$ sidered to be secondaries.

In the tests that are described, care was taken to check that there were no EMG signs of contractions occurring in the receptor bearing $\overline{\bar{y}}$ muscles. When such contractions occurred, either unintentionally or upon command to push or pull against resistance, the firing rate of the spindle afferent fibres, primaries as well as secondaries, was usually enhanced (Fig. 2, IV).응 However, spindle unloadings were seen when contractions occurred in neighbouring muscles, synergistic to the receptor bearing one (Fig. 3, N AIII). Such unloadings were most prominent when joint movements were allowed to occur as a result of the synergistic contractions, but the phenomenon was also present in isometrio contractions when no joint movements occurred. Because of the difficulties in activating selectively 
only one particular muscle in a group of synergists, the spindle responses to isometric contractions were sometimes of a composite nature. Temporary unloading effects from contractions in neighbouring muscles were probably responsible for the decrease in spindle firing sometimes seen at the start of a voluntary contraction (Fig. 3, BI).

In a few experiments a mechanical vibrator with a stroke amplitude of $1.5 \mathrm{~mm}$ was applied over the receptor bearing muscle or its tendon. When the muscle was kept in a stretched position

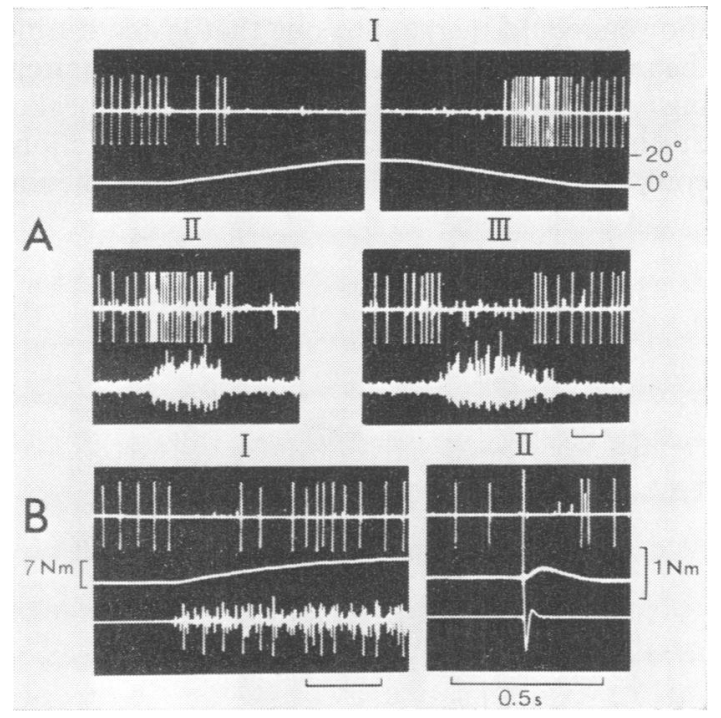

FIG. 3 Further examples of Ia spindle responses (upper trace) to passive changes of muscle length and muscle contraction. Unit $\mathrm{A}$, located in index finger portion of finger flexors, shows a sustained resting discharge when fingers are fully extended. I: spindle unloading by passive flexion of metacarpophalangeal joints (angle in bottom trace). II: acceleration of spindle discharges by voluntary isometric flexion of index finger (finger flexor EMG in bottom trace). III: spindle unloading by voluntary isometric flexion of middle finger (finger flexor EMG in bottom trace). Unit B located in the anterior tibial muscle. I: isometric dorsiflexion of foot, presumably aided by contraction in long toe extensors, causes initial unloading of the spindle (torque and tib. ant. EMG in middle and bottom traces). II: response to an electrically elicited muscle twitch in the anterior tibial muscle (torque and tib. ant. EMG in middle and bottom traces). some of the Ia afferent fibres could be driven by vibration frequencies of up to $100 \mathrm{~Hz}$. When the muscle was passively shortened, however, the vibrator became a less potent stimulator, as the firing frequency often fell to levels which were subharmonics of the mechanical oscillations (Fig. 2, V).

SPINDLE DISCHARGES IN VOLUNTARY ALTERNATING MOVEMENTS As seen in EMG recordings during fast voluntary finger- and foot movements of the alternating type, the flexor contractions usually start at the end of the extension phases and the extensor contractions at the end of the flexion phases, each fast contraction apparently generating energy to change the direction of the movement. Spindle records obtained during such active alternating movements showed early stretch discharges similar to those seen in passive alternating movements but also discharges during the later parts of the stretch phases, partly coinciding with the eccentric muscle contractions. An example is shown in Fig. 4A where a primary spindle afferent fibre from a finger flexor muscle is coactivated with the flexor EMG during the extension phases.

Particular attention was paid to contractions which initiated a series of alternating movements and which differed from the succeeding ones by not starting during muscle stretch and therefore not producing deceleration of movement. Discharges in primary spindle afferent fibres were also observed during these initial ballistic contractions which were isometric in the sense that the EMG burst had ended before the movement started (Fig. 4B). Both in finger flexor and anterior tibial contractions of this type there was a small time lag between the EMG burst and the spindle discharge, the latter occurring mainly during the short period of active rise of tension before the movement. The number of unitary spindle impulses initiated during the contraction phase seldom exceeded five to six. The number varied between different units and for each unit it also varied from one movement to the next. On repetition of rapid finger flexor movements, a flexor spindle occasionally failed to respond during the contraction and in a few instances the contractioninstead of activating the spindle-caused a short pause in a pre-existing static spindle dis- 


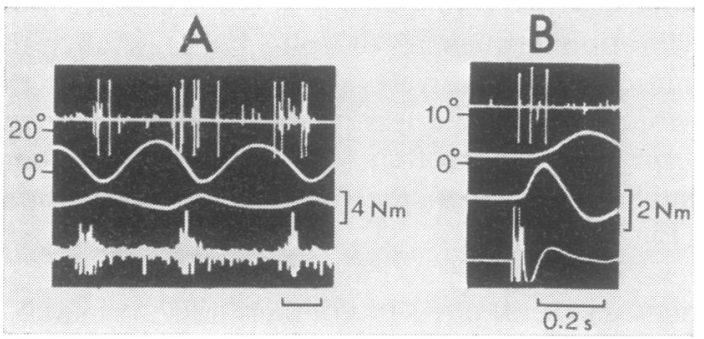

FIG. 4 A: Discharges of a Ia afferent unit from the finger flexors during voluntary fast alternating finger movements (moving fingers attached to supporting plate). B: Discharges of a Ia afferent unit from the anterior tibial muscle during voluntary fast dorsiflexion of foot (first contraction in a series of alternating foot movements). Moving foot attached to supporting plate. Upper trace: nerve records. Second trace: angle in the metacarpophalangeal (A) or ankle joint (B). Third trace: torque. Bottom trace: EMG from finger flexor (A) and anterior tibial muscles (B).

charge. In such instances, the contraction discharge could usually be restored by instructing the subject to make 'clean' finger flexor movements without undue involvement of neighbouring forearm muscles.

SPINDLE DISCHARGES IN ISOMETRIC ALTERNATING MUSCLE CONTRACTIONS In these tests the subject tried to make fast alternating or single rapid movements, but, since the supporting plate was fixed, no muscle length changes occurred beyond those allowed by the viscoelastic events. Figures 5 and 6 show some examples of afferent Ia fibre responses during rapid voluntary contractions of this type. As in electrically induced twitches, the spindles usually fired during the relaxation phases, but in the voluntary twitches spindle discharges were also seen during the phases of active tension rise. The discharge pattern of individual units, however, was seldom identical from one voluntary twitch to the next, probably depending on random small variations in the spatial distribution of the extrafusal twitches, relative to the site of the receptor. Thus, during alternating contractions it sometimes happened that a unit, initially firing during both rising and falling phases, suddenly altered its firing pattern and started to respond only during one of these phases. In Fig. 5 all three units show examples of this behaviour, but the change in pattern is best seen for the unit in Fig. 5A. Some of the units, which showed a more predictable biphasic firing pattern, responded predominantly during the rising phase, others during the falling phase of the twitch (cf Fig. 6A and B). As was the case during passive stretch movements, fewer impulses were discharged if the muscle were initially short than if it were held in a stretched position (Fig. 6C).

As a rule the biphasic firing pattern of a unit was easy to distinguish, but when the subjects were simulating a fast tremor the two impulse showers could merge into one that lasted during the major part of the twitch. Thus, when monitoring the nerve activity in the loudspeaker during such tests, the recurrent contractions could be associated with bursts of neural

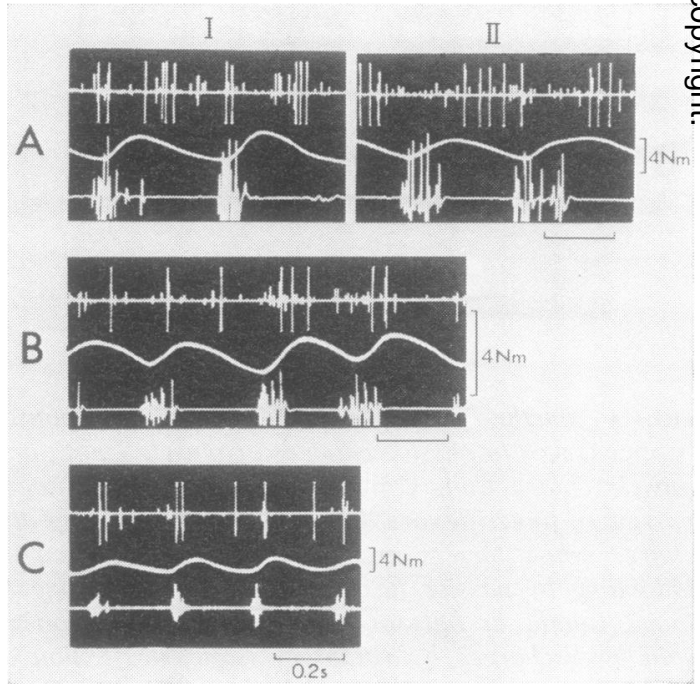

FIG. 5 Examples of contraction and relaxation discharges in Ia afferent units during fast active alternating contractions, isometrically recorded. Unit A and B located in anterior tibial muscle (same units as in Fig. $I B$ and $C$ respectively). Unit $C$ in the finger flexors (same as in Fig. 1A). Upper traces: nerve records. Middle traces: torque. Bottom traces: EMG from the anterior tibial (A and $\mathrm{B})$ and the finger flexor (C) muscles. 


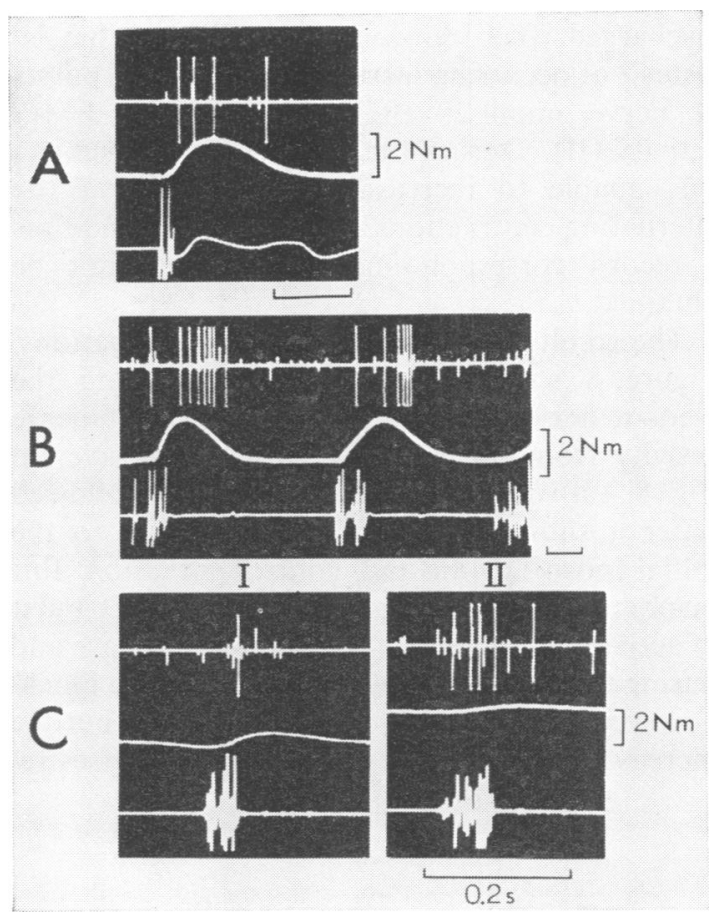

activity at the same frequency as the contractions or at double that frequency.

EFFECT OF LIDOCAINE BLOCK ON SPINDLE RESPONSES On two occasions, unitary Ia fibre recordings from the anterior tibial nerve were maintained long enough to test the effect of a partial lidocaine nerve block (proximal to the recording site) on spindle firing during voluntary contractions. The results were similar on the two occasions in showing that the block eliminated the contraction discharges in the Ia afferent

FIG. 6 Examples of contraction and relaxation discharges in Ia afferent units during single fast isometric contractions of receptor bearing muscles. Unit $\mathrm{A}$ and $\mathrm{B}$ located in anterior tibial muscle (unit $B$ the same as in Fig. $1 B$ and Fig. 5A), unit $\mathrm{C}$ in the finger flexors. In CI the contraction is made at short and in CII at maximal muscle length (fingers flexed $70^{\circ}$ and fully extended respectively). Upper traces: nerve records. Second traces: torque. Bottom traces: EMG from the anterior tibial (A and $\mathrm{B}$ ) and the finger flexor (C) muscles.

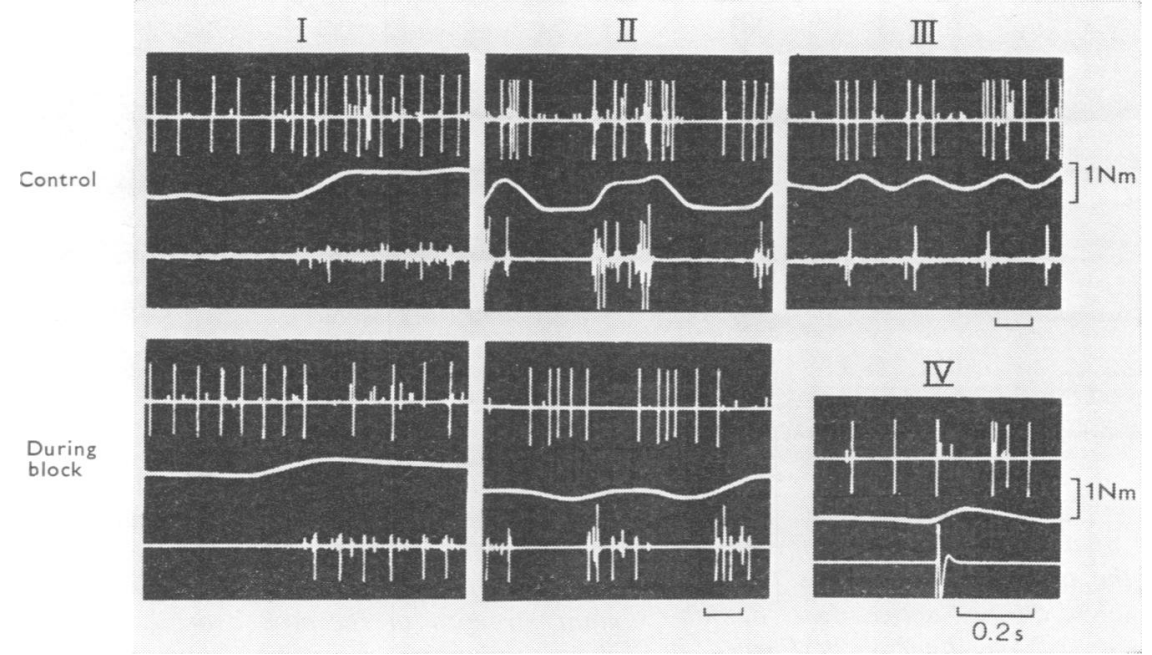

FIG. 7 The effect of a partial lidocaine block of the peroneal nerve on the discharges of a Ia afferent unit from the anterior tibial muscle during maintained isometric contraction (I) and slow alternating isometric contractions (II). The control run in III shows rapid alternating contractions which the subject was unable to make during the block. In IV the unit shows the typical Ia behaviour during an electrically elicited muscle twitch (before the block). The nerve block was achieved by injecting $1 \%$ lidocaine around the nerve in the popliteal fossa, proximal to the nerve recording site at the fibular head. Upper traces: nerve records. Middle traces: torque. Bottom traces: $E M G$ from the anterior tibial muscle. 
fibres. Figure 7 illustrates the findings when recording from a single Ia afferent unit during isometric conditions, the foot being fixed in $10^{\circ}$ plantar flexion. In the control situation the unit showed a low frequency static discharge and as usual a sustained voluntary contraction of the anterior tibial muscle led to an increase in the firing rate (I). During slow alternating contractions, bursts of impulses occurred, mainly coinciding with a period of increased tensionthat is, in parallel with the EMG activity, and were followed by short silent periods (II). Contraction responses of similar type were seen when the contraction frequency increased to $3-4 \mathrm{~Hz}$ (III). During the nerve block the response pattern was altered. Instead of increasing the firing rate of the unit, a sustained voluntary contraction caused a decrease of the firing frequency (I). During slow alternating contractions the unit paused during the tension increase and

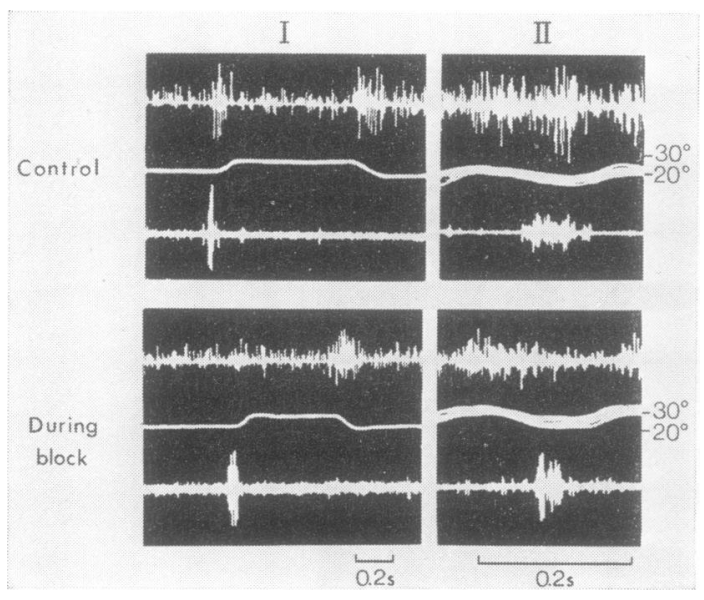

FIG. 8 The effect of a partial lidocaine block of the median nerve on the multi-unit discharges from the finger flexor muscles during active fast flexionextension movements of the fingers, performed in single steps (I) or in a smooth alternating fashion (II). In II four consecutive oscilloscope sweeps have been superimposed. The nerve block was achieved by injecting $1 \%$ lidocaine around the nerve $10 \mathrm{~cm}$ proximal to the nerve recording site at the elbow. Upper traces: nerve records. Middle traces: angle in the metacarpophalangeal joints. Bottom traces: finger flexor EMG. discharged when tension was reduced-that is, instead of occurring largely in parallel, the bursts of nerve impulses alternated with the EMG activity (II). During the anaesthesia the subject was unable to increase the frequency of the alternating contractions above $1-2 \mathrm{~Hz}$-and thus a record corresponding to III could not be obtained.

The results were similar in isotonic situations. Figure 8 shows the results of blocking the median nerve while recording multi-unit nerve activity from the deep finger flexor muscle. In Fig. 8, I a voluntary finger flexion was followed (after a short while) by a rapid return to the initial position. In the control situation this manoeuvre was associated with a distinct burst of activity both during the contraction phase and during the stretch phase. During the nerve block, however, no contraction bursts occurred but the increase of activity during the stretch phases re-

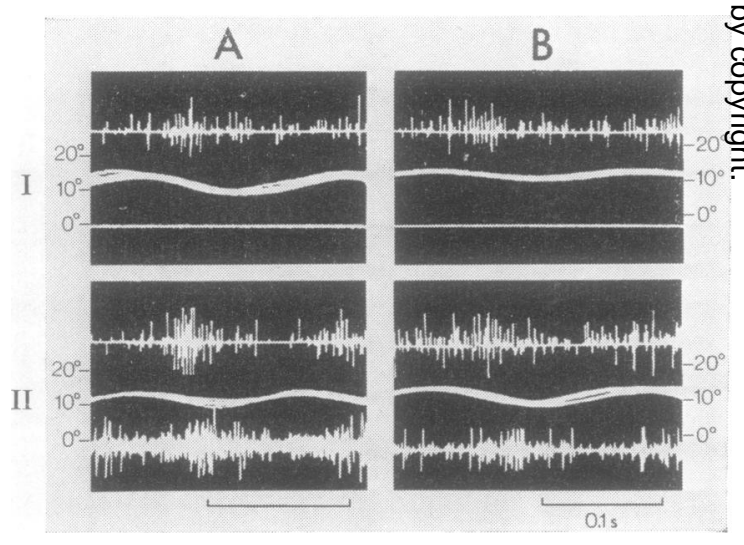

FIG. 9 Afferent multi-unit stretch discharges and motor events during passive alternating movements when the subject was instructed to relax (I) and when instructed to maintain voluntary contraction of constant strength in receptor bearing muscles (II). A shows the nerve recording from the median nerve fascicle innervating the finger flexor muscles and $\mathrm{B}$ from peroneal nerve fascicle innervating the anterior tibial muscle. Four consecutive oscilloscope sweeps superimposed. Upper traces: Nerve records. Middle traces: Angle in the metacarpophalangeal (A) and ankle joints (B). Lower traces: EMG from the finger flexors (A) and the anterior tibial muscles (B). Note involuntary increase of contraction strength during stretch phases in II. 
mained seemingly unaltered. Figure 8, II shows superimpositions of four rapid alternating contractions when the subject was instructed to simulate fast tremor. During the control runs an increased multi-unit activity is seen starting at the beginning of the stretch phase and lasting until the end of the EMG activity, and with the most prominent part of the response occurring during the EMG-burst. When the nerve was blocked, this latter part of the response disappeared and only the increase of activity corresponding to the early stretch phase remained.

REFLEX EFFECTS OF Ia FIBRE DISCHARGES DURING ALTERNATING MOVEMENTS As shown in Fig. 1, the spindle discharges evoked by passive alternating movements were not followed by any reflex responses in the EMG. All subjects showed this ability to remain relaxed in the receptor bearing muscles, even during alternating movements at the rate of $10 \mathrm{~Hz}$ (Fig. 9, I). However, as shown by Hoffmann (1922), it is not possible to maintain a voluntary contraction of constant strength in muscles actively resisting such rapid length changes. This was repeatedly confirmed in the present study. The superimposed traces in Fig. 9, II illustrate that, in spite of the subject's attempt to keep the muscle contractions constant, the EMG activity rises at the end of the stretch phases and wanes during the shortening phases. The EMG bursts were preceded by neural stretch discharges, the latency between the two events being $15-20 \mathrm{~ms}$ in the arm and slightly longer in the leg.

\section{DISCUSSION}

ALPHA-GAMMA COACTIVATION IN RAPID MOVEMENTS A major technical problem in studies of the present type is to ascertain that the electromyograms derive from those particular muscles in which the receptors are located. Unless needle or wire EMG electrodes are used mistakes can easily be made, especially in dealing with the anatomically complicated forearm muscles. During active alternating finger movements, it was observed, for instance, that flexor carpi muscles were sometimes acting out of phase with the finger flexor muscles, supposedly subserving a stabilizing function during the extension phases; and it was of the utmost importance for the interpretation of the results that in such instances the flexor carpi EMG signals were not misjudged as originating from the finger flexors.

The coexcitation of the spindle primary endings described in the present study is difficult to explain in ways other than in terms of a fusimotor outflow occurring in parallel with and directed towards the same muscles as the outflow in the skeletomotor nerve fibres. The alternatives would be ephaptic transmission or excitatory effects resulting from mechanical events in the motor units lying in the immediate vicinity of the receptor (Granit et al., 1959). There are two main reasons why these alternatives seem most unlikely: (1) The spindle coexcitation was seen only during voluntary contraction, not during electrically induced muscle twitches. (2) It was not seen in voluntary contractions performed during partial lidocaine block of the motor nerve.

DELAY OF SPINDLE COEXCITATION In agreement with Vallbo's observations (1971), it was found that in rapidly rising isometric contractions the spindle acceleration starts after the appearance of the first EMG signals, implying that such contractions are initiated by the direct route to the muscles, any potential gamma loop contribution to the contraction being delayed at least 20 $\mathrm{ms}$ (which is the approximate latency of the stretch reflex in the finger flexors).

As originally shown by Kuffler et al. (1951), the fusimotor induced spindle acceleration becomes more potent and starts earlier the more the muscle is stretched, and in the present study we noted a similar potentiating effect of muscle stretch upon the contraction discharge from the spindles (Fig. 6C). In the most shortlasting isometric contractions ( $<50 \mathrm{~ms})$, many of the spindle impulses appeared too late to influence the motor event-that is, they appeared after the EMG bursts had ended (Fig. 6A). When the contractions lasted more than $50 \mathrm{~ms}$, however, and in particular when such contractions occurred in stretched muscles, the spindle acceleration started sufficiently early to permit a reflex influence on motor unit firing in the later part of the contraction phases-for example, Fig. 6C.

It was more difficult to evaluate the fusimotor influence upon the spindle discharges in fast alternating movements when the muscle length 
changed continuously during the contraction phases. However, a comparison of Fig. 1A with Fig. 4A and Fig. 9, I with Fig. 8, control II illustrates the differing spindle discharge patterns in passive as compared with active alternating movements: in the active movements, the early stretch discharge is succeeded by a second discharge partly coinciding with the EMG burst. As judged by the superimposed multi-unit records in Fig. 8, control II the spindle accelerations accompanying the eccentric contractions started at least $50 \mathrm{~ms}$ before the contractions ended-that is, early enough for the later part of the contraction to receive some gamma loop support.

EFFECT OF CONTRACTIONS IN DISTANT AND NEIGHBOURING MUSCLES According to Vallbo (1970), the fusimotor outflow tends to be directed to the same muscles or muscle portions as the skeletomotor outflow, not to the spindles outside this region. Our findings support this notion. It should be emphasized, however, that in voluntary movements the skeletomotor outflow is not restricted to the prime movers. There are often postural adjustments taking place in distant muscle groups, and, as shown by Burg et al. (1974), spindle accelerations can be seen in such distant muscles not primarily engaged in the voluntary motor act. However, we were not able to confirm the notion of a fusimotor outflow directed to distant muscles in which there are no concomitant EMG signs of skeletomotor activity (Hagbarth et al., 1975).

Contractions in muscles lying close to the one in which the receptors were located often led to a decrease of the spindle impulse rate, and, in agreement with Vallbo, we interpreted this phenomenon as a mechanical unloading. It may be assumed that when a group of neighbouring synergists are coactivated in a voluntary contraction the spindle excitation in each of them tends to be reduced by the unloading effect from the others. Thus, in many of the spindle coexcitations observed in the present study, the firing rate of the units might well have been higher had the subjects been able to activate selectively only the receptor bearing muscle.

FUNCTIONAL ROLE OF SPINDLE DISCHARGES IN RAPID ALTERNATING MOVEMENTS Evidence is accumulating that not only fast alternating movements like those dealt with in the present study but also movements like walking, running, swimming, etc, are centrally patterned and may represent types of stored motor programmes (De Long, 1971) that can operate without phasic sensory feed-back. Still, it is reasonable to assume that sensory feed-backs can help to make the movements run in a smoother fashion-for instance, by adjusting the phasing and the strength of the contractions to existing external loads.

The observations that a spindle coexcitation occurs and that there is time enough for the gamma loop to participate in the rapid contractions during fast alternating movements do not necessarily imply that the loop is essential for the motor performance. The autogenetic excitatory effect of the contraction discharges in the Ia fibres may, for instance, be balanced out by autogenetic inhibitory effects from Golgi tendon organs, or the spinal paths mediating autogenetic excitation in movements of this type may be closed by supraspinal action. A stretch reflex modulation of the EMG interference patterte occurs, however, when passive rapid movements are performed against active resistance (Fig. 9 라 II) and it has also been demonstrated that, during slow voluntary movements, minute unintentional irregularities in the speed of movement modulate the strength of the motor outflow through dynamic variations in spindle discharge (Vallbo, 1974). Assuming that the motoneurone pools are in a similar way responsive to dynamic changes in spindle input during the contraction phases in fast alternating movements, the gamma loop may serve to adjust the strength of the contractions to the external momentum that has to be overcome in each movement cycle. Since the loop also has inhibitory projections to antagonistic motor neurones, it may also help to keep flexors and extensors working in a regular reciprocal fashion.

It has been proposed that programmes of motor behaviour for the voluntary production of rapid alternating movements may be stored in somatosensory cortex (Alberts, 1972) and recent studies indicate that the cerebellum plays an important role in governing the correct timing and duration of muscular bursts in such movements (Conrad and Brooks, 1974). Considering 
the richness of muscle afferent projections to the cerebellum (Oscarsson, 1965) and the evidence for cerebellar control of both static and dynamic fusimotor fibres (Granit, 1970) it may be suggested that the muscle spindle discharges from the working muscles, as recorded in the present study, provide information which the cerebellum needs to calculate the timing and coordination of rapid programmed movements.

The suggestions presented above agree with the outcome of the lidocaine tests, which showed that both rapid and sustained voluntary contractions can be performed without concomitant fusimotor activation of the spindles. However, in all three lidocaine tests the subjects had obvious difficulties in maintaining constant high speeds in their movements. As in positive diadochokinesis tests, the regular rhythm was often lost and the movements became more jerky and irregular.

\section{REFERENCES}

Alberts, W. W. (1972). A simple view of Parkinsonian tremor. Electrical stimulation of cortex adjacent to the Rolandic fissure in awake man. Brain Research, 44, 357-369.

Burg, D., Szumski, A. J., Struppler, A., and Vehlo, F. (1974). Assessment of fusimotor contribution to reflex reinforcement in humans. Journal of Neurology, Neurosurgery, and Psychiatry, 37, 1012-1021.

Conrad, B., and Brooks, V. B. (1974). Effects of dentate cooling on rapid alternating arm movements. Journal of Neurophysiology, 37, 792-804.

De Long, M., II (1971). Central patterning of movement. Neurosciences Research Program Bulletin, 9, 10-30.
Granit, R., Pompeiano, O., and Waltman, B. (1959). The early discharge of mammalian muscle spindles at onset of contraction. Journal of Physiology, 147, 399-418.

Granit, R. (1970). The Basis of Motor Control, pp. 225-247. Academic Press: London.

Green, D. G. (1967). A transistor instantaneous frequency meter. Medical and Biological Engineering, 5, 387-390.

Hagbarth, K.-E., and Vallbo, A. B. (1969). Single unit recordings from muscle nerves in human subjects. Acta Physiologica Scandinavica, 76, 321-334.

Hagbarth, K.-E., Hongell, A., Hallin, R. G., and Torebjörk, H. E. (1970). Afferent impulses in median nerve fascicles evoked by tactile stimuli of the human hand. Brain Research, 24, 423-442.

Hagbarth, K.-E., Wallin, B. G., Burke, D. and Löfstedt, L., (1975). The effect of Jendrassik's manoeuvre on muscle spindle activity in man. Journal of Neurology, Neurosurgery, and Psychiatry. (In press.)

Hoffmann, P. (1922). Untersuchungen über die Eigenreflexe (Sehnenreflexe) menschlicher Muskeln, p. 69. Springer: Berlin.

Kuffler, S. W., Hunt, C. C., and Quilliam, J. P. (1951). Function of medullated small-nerve fibers in mammalian ventral roots: efferent muscle spindle innervation. Journal of Neurophysiology, 14, 29-54.

Oscarsson, O. (1965). Functional organization of the spinoand cuneocerebellar tracts. Physiological Reviews, 45, 495522.

Vallbo, Å. B. (1970) Discharge patterns in human muscle spindle afferents during isometric voluntary contraction. Acta Physiologica Scandinavica, 80, 552-566.

Vallbo, Å. B. (1971). Muscle spindle response at the onset of isometric voluntary contractions in man. Time difference between fusimotor and skeletomotor effects. Journal of Physiology, 218, 405-431.

Vallbo, A. B. (1974). Human muscle spindle discharge during isometric voluntary contractions. Amplitude relations between spindle frequency and torque. Acta Physiologica Scandinavica, 90, 319-336.

Wallin, B. G., Hongell, A., and Hagbarth, K.-E. (1973). Recordings from muscle afferents in parkinsonian rigidity. In New Developments in Electromyography and Clinical Neurophysiology, vol. 3, pp. 263-272. Edited by J. E. Desmedt. Karger: Basel. 